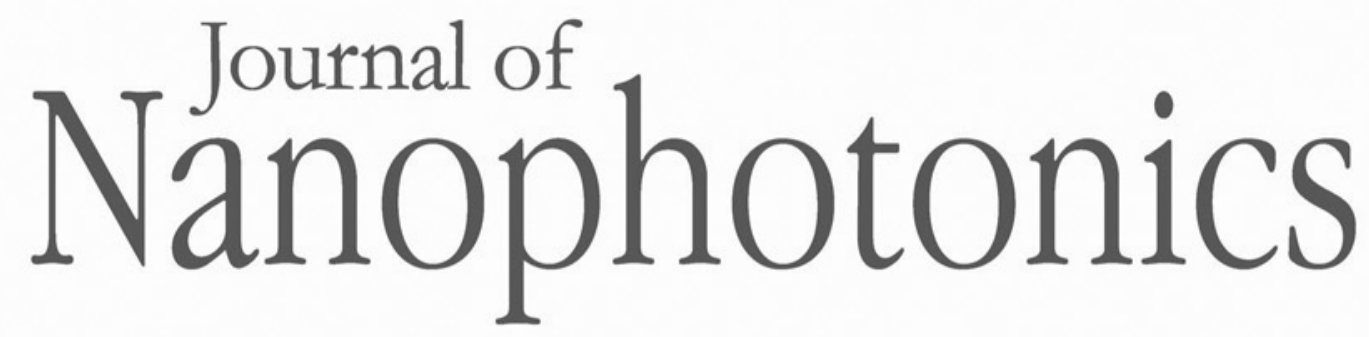

\title{
Calculation of reflectivity spectra for semi-infinite two-dimensional photonic crystals
}

\author{
Mirsaeid Sarollahi \\ Stephen J. Bauman \\ Jonathan Mishler \\ Joseph B. Herzog
}




\title{
Calculation of reflectivity spectra for semi-infinite two-dimensional photonic crystals
}

\author{
Mirsaeid Sarollahi, ${ }^{a, \dagger}$ Stephen J. Bauman, ${ }^{\text {a, }}$ 'Jonathan Mishler, ${ }^{b}$ and \\ Joseph B. Herzog ${ }^{\mathbf{a}, \mathbf{b}, *}$ \\ ${ }^{a}$ University of Arkansas, Microelectronics-Photonics Program, 731 West Dickson Street, \\ Fayetteville, Arkansas 72701, United States \\ ${ }^{b}$ University of Arkansas, Department of Physics, 825 West Dickson Street, PHYS 226, \\ Fayetteville, Arkansas 72701, United States
}

\begin{abstract}
This work involves the development of a finite-element method model to examine the optical properties of two-dimensional photonic crystals (PCs). The model is capable of studying the effect of a finite number of periods in a PC structure. The new design minimizes computational resources by modeling a PC with one infinite dimension with periodic boundary conditions while modeling the second with finite dimensions. This allows for calculation of transmission and reflection spectra across the PC structure. A finite difference frequency domain (FDFD) model has been created for calculation of the photonic band structure. This is compared with the reflection spectra obtained through the reflection model and is found to closely match. The reflection model capabilities are demonstrated by calculating the reflection spectrum for various parameters: period length, number of periods, incident light polarization, and material properties. Effects of varying these parameters are demonstrated. For example, the reflectivity of a GaAs/Air PC was found to reach greater than 95\% when the PC has 10 periods; it exceeds 99\% with 13 periods and reaches $99.9 \%$ at 15 periods. (C) The Authors. Published by SPIE under a Creative Commons Attribution 3.0 Unported License. Distribution or reproduction of this work in whole or in part requires full attribution of the original publication, including its DOI. [DOI: 10.1117/1.JNP.10.046012]
\end{abstract}

Keywords: photonic crystal; finite difference frequency domain; photonic band gap.

Paper 16119P received Jul. 22, 2016; accepted for publication Oct. 24, 2016; published online Nov. 16, 2016.

\section{Introduction}

Photonic crystals (PCs) are periodic structures of dielectric material that can reflect light with high efficiency due to optical interference effects; this allows PCs to bend and control light with high precision. PCs can be designed to have forbidden optical frequency bands that are known as photonic band gaps (PBGs). In addition to commercially available low-loss PC fibers, other applications of PCs include low-threshold lasers, ${ }^{1}$ single-mode light emitting diodes, ${ }^{2,3}$ Bragg mirrors, ${ }^{4-6}$ optical filters, ${ }^{7-9}$ and efficient planar antennas. ${ }^{10}$ PCs two-dimensional (2-D) periodicity can guide light in a plane. The propagated light inside the PC structure is affected mainly by material properties (refractive index) and the period size of the crystal structure. In addition, the direction of light polarization and wavelength has a significant effect on reflectivity of a PC waveguide. ${ }^{11-13}$

Various numerical computation methods exist for studying the optical properties of PCs. Transfer matrix methods, ${ }^{14,15}$ finite-element methods (FEMs), ${ }^{16-20}$ finite difference time domain, ${ }^{21-28}$ finite difference frequency domain (FDFD) methods, ${ }^{29-32}$ and plane-wave expansion methods ${ }^{33-36}$ have been developed over the past few decades for this purpose. When modeling 2-D PCs, infinitely periodic boundary conditions are often used in both dimensions, ${ }^{26,30,33,37}$ or the models can be finite in both directions. ${ }^{38-40}$ Yet, it is impossible to fabricate a PC with an infinite number of periods, and it is challenging and expensive to fabricate highly

*Address all correspondence to: Joseph B. Herzog, Email: jbherzog@uark.edu

${ }^{\dagger}$ Authors have contributed equally to this work. 
uniform PCs with many periods. Therefore, investigating the effects of varying the number of periods in a PC is important for optimally fabricating them. The model introduced here can help precisely determine how many periods are needed to achieve the desired reflectivity for a given incident wavelength. Others have made use of semi-infinite models to study PC properties, but none of these specifically investigates the precise effect on the number of periods relative to reflection spectra. ${ }^{41-43}$ This work introduces a useful model to calculate the reflectivity spectrum for a 2-D PC with a finite number of periods in one dimension and an infinite periodicity in the perpendicular direction. The effects of varying the situational parameters (polarization, material properties, number of periods, and so on) can be easily investigated, as demonstrated in this work. Incorporation of wavelength-dependent dielectric functions is a key benefit of the model. The results are compared to the photonic band structure calculated through a FDFD model to compare the reflectivity spectrum with the photonic band structure.

\section{Reflection Model}

Reflection spectra from various PC configurations were calculated using a FEM model (geometry created and discretized through COMSOL Multiphysics). The model, shown in Fig. 1(a), is a semiperiodic lattice of circles (cylinders, if the infinite $z$-dimension is considered) of one material within a matrix of another material. The $y$-dimension has a finite number of periods, whereas in the $x$-direction, it is modeled to be infinitely periodic by assigning periodic boundary conditions. This model has been developed to study the effects of variable geometric and material parameters, especially the number of periods and the period size, on the reflection spectra of light incident on the structure in the negative $y$-direction. The materials chosen were GaAs due to its relatively high refractive index in the visible range, $n_{\mathrm{H}} \approx 3.5$ to 5 , and air with $n_{\mathrm{L}}=1.0{ }^{44}$ The large $n_{\mathrm{H}} / n_{\mathrm{L}}$ ratio improves the overall reflectivity of the PC. ${ }^{45}$

The model consists of a vertical unit cell of a square crystal lattice with periodic boundary conditions in one dimension ( $\pm x$ directions). The number of periods in the $y$-direction, $N$, is varied, as shown in Fig. 1(a). Light is incident in the negative $y$-direction. Materials studied in this work include a $\mathrm{GaAs}, \mathrm{Si}$, or $\mathrm{SiO}_{2}$ slab with air holes, and $\mathrm{GaAs}$ posts surrounded by air (the model extends infinitely in the $z$-direction). The thickness of the simulation space is a function of the number of periods inside the unit cell so that the distance between the incident light port and the $\mathrm{PC}$ is always four times the period $(4 P)$. In this model, the incident light is polarized in either the transverse electric (TE) or transverse magnetic (TM) direction, with respect to the $z$-axis.

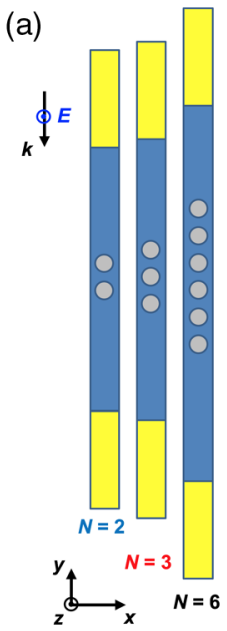

(b)

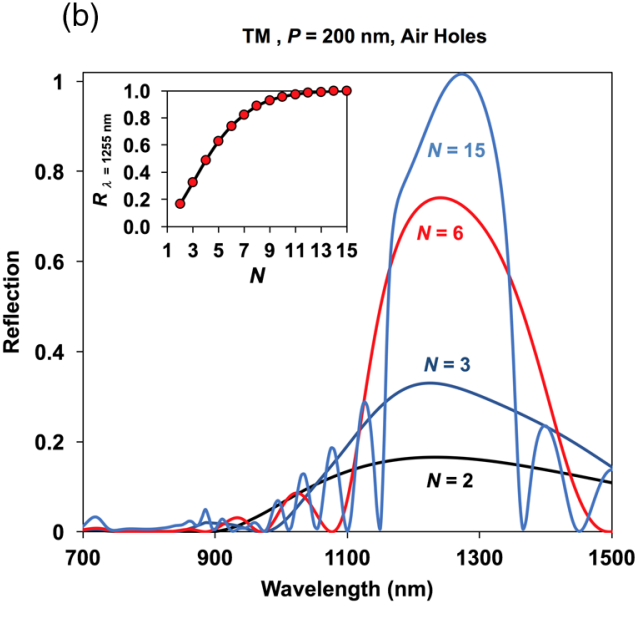

Fig. 1 (a) Depiction of the model geometry. Perfectly matched layers are shown in yellow at the top and bottom of the geometry. The PC was modeled as a GaAs slab with air holes. The region shown was modeled to have left and right edges that are infinitely periodic. The number of vertical periods $(N)$ can be changed as shown. The incident light direction $(k)$ and polarization direction $(E)$ are labeled relative to the model. (b) Plot of reflection spectra for different values of $N$. The PBG is seen with a central peak around $1255 \mathrm{~nm}$. The inset plots the maximum reflection versus period. 


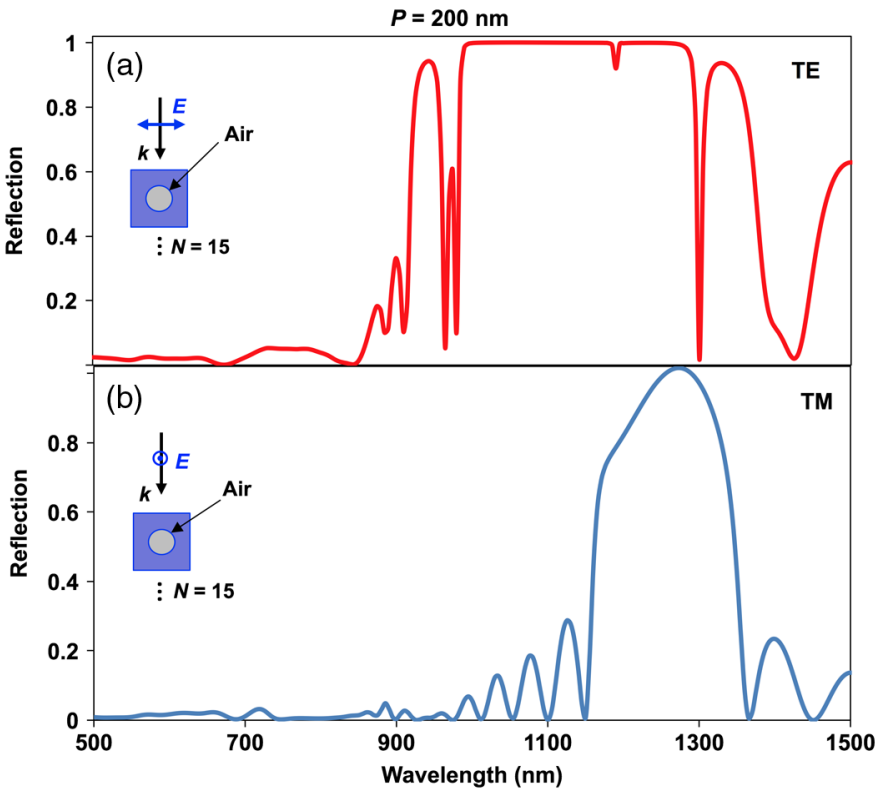

Fig. 2 Effect of polarization on the PBG. Reflection spectrum for (a) TE mode and (b) TM mode with $N=15$ and $P=200 \mathrm{~nm}$.

Figure 1(b) shows how the reflection spectrum changes as a function of the number of periods, $N$, in the unit cell. The results were calculated for air holes in GaAs with a constant period (center-to-center distance) of $P=200 \mathrm{~nm}$. Incident light was polarized in the $z$-direction, which is also known as the TM mode for PCs. ${ }^{46}$ As $N$ was increased, the reflection peak was found to increase for PBG wavelengths, as expected. The inset plots the maximum reflection at the PBG as a function of $N$. At $1255 \mathrm{~nm}$, the reflection magnitude for $N=2$ is $16.4 \%$, and for $N=15$, it reaches $99.9 \%$. The reflectivity reaches a value $>95 \%$ at $N=10$ and exceeds $99 \%$ when $N=13$. The work by Abdulhalim ${ }^{47}$ demonstrates the same trend of increasing reflection for an increasing number of periods for stacked anisotropic wave plate layers.

Figure 2 shows the polarization dependence of the PC reflection spectrum. The constant parameters for this test were $N=15$ and $P=200 \mathrm{~nm}$ with air holes in GaAs. Only the polarization was changed, with the incident light being polarized in $x$ (TE mode) and $z$ (TM mode), as shown in Figs. 2(a) and 2(b), respectively. The width of the PBG was found to be greater for the TE mode compared to the TM mode. The PBG wavelength range was $\sim 500 \mathrm{~nm}$ for the TE mode and $300 \mathrm{~nm}$ for the TM mode; for TE polarization, the reflectivity is near $100 \%$ across the entire width of the PBG.

The effects of varying material properties on the PBG were also investigated, as seen in Fig. 3. GaAs posts in air [Fig. 3(a)] exhibit a wider PBG than air holes in GaAs [Fig. 3(b)]. The width of the PBG wavelength range for GaAs posts was $\sim 500 \mathrm{~nm}$, while for air holes, it was $\sim 300 \mathrm{~nm}$. Air holes in GaAs show higher reflectivity $\left(R_{\max }=99.9 \%\right)$ than the peak value for GaAs posts in air $\left(R_{\max }=87.8 \%\right)$ when both unit cells contain $N=15$ periods. For GaAs posts, the PBG has a center wavelength near $725 \mathrm{~nm}$, and for air holes, the PBG center wavelength was near $1255 \mathrm{~nm}$.

This model was lastly used to plot the PBG peak shift as a function of the PC period. The resulting reflection spectra are shown in Fig. 4 in a waterfall plot, where $P$ varies from 200 to $300 \mathrm{~nm}$. As $P$ is increased, the PBG central wavelength is redshifted. This trend is plotted in the inset of Fig. 4 for the wavelengths of peak reflection, $\lambda_{\text {peak }}$; it shows that $\lambda_{\text {peak }}$ increases linearly with $P$, where $\lambda_{\text {peak }}=5.96 P+81 \mathrm{~nm}$.

A small PBG can be seen for $P=250$ to $300 \mathrm{~nm}$. The gap shifts to lower wavelengths as a function of $P$, similar to the behavior of the main higher-wavelength PBG region. Once it shifts to wavelengths $<875 \mathrm{~nm}$, the reflectivity drops to zero because at this point, the photon energy exceeds the bandgap of GaAs, permitting light to be absorbed into the GaAs. The presence of this phenomenon helps to confirm the validity of the results. Using a material with a larger 


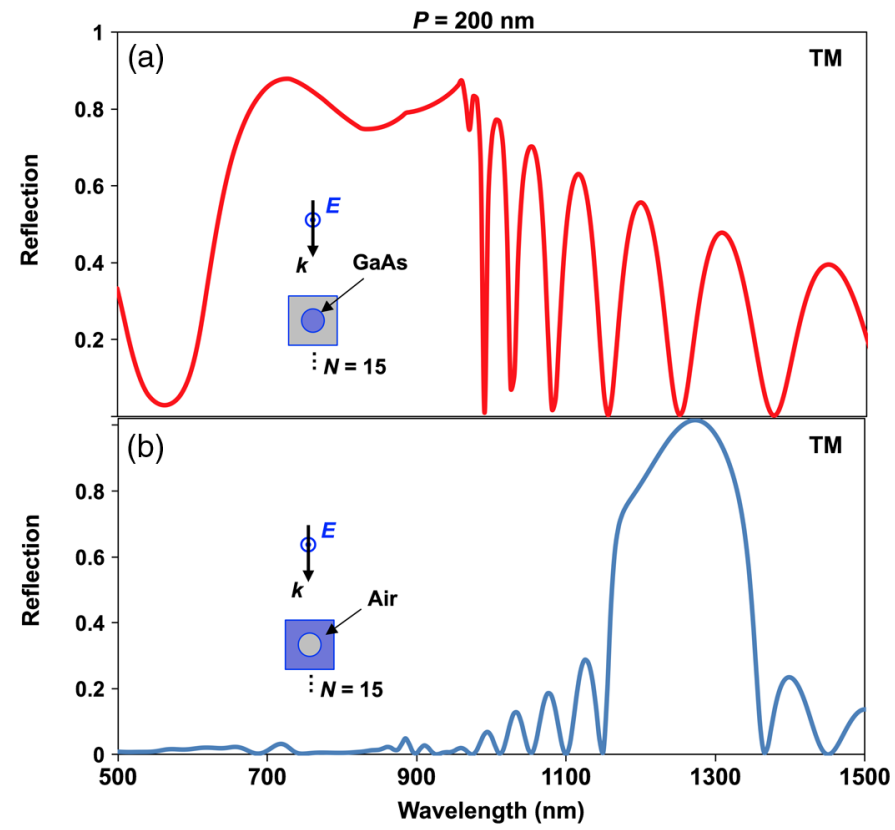

Fig. 3 Effects of material properties on PBG. Reflection spectrum for a PC with (a) GaAs posts in air and (b) air holes in GaAs. Constant parameters are TM polarization, $N=15$ and $P=200 \mathrm{~nm}$.

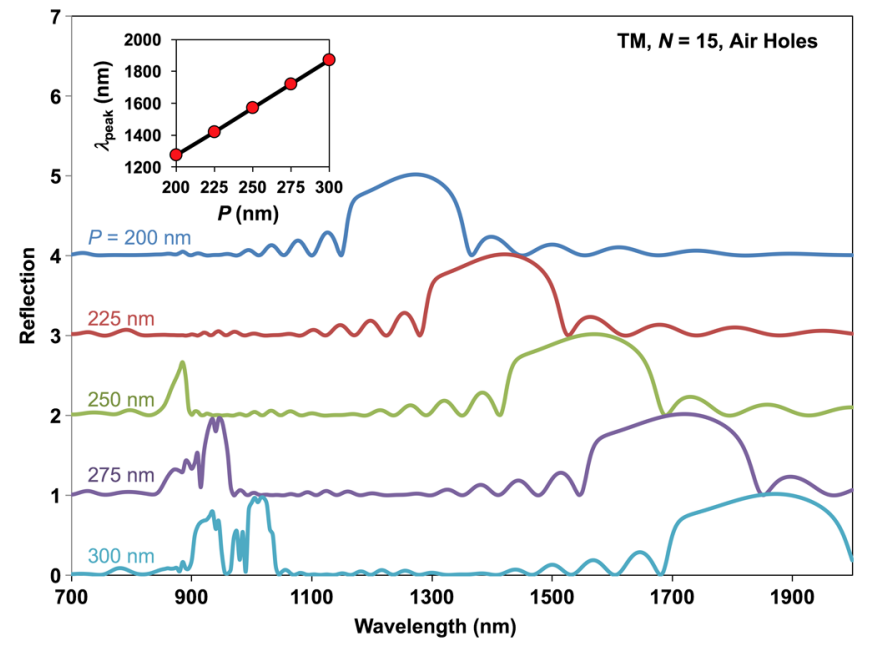

Fig. 4 Reflection spectra for varying PC period widths $(P)$. Constants here are $N=15$, TM polarization, and air holes in a GaAs surrounding medium.

bandgap can extend the PBG to lower wavelengths; however, the bandgap width is typically inversely proportional to the refractive index of a given material. ${ }^{48}$ This reduces the reflectivity, as $R$ increases with $\left(n_{\mathrm{H}} / n_{\mathrm{L}}\right)^{2 N}$; therefore, a larger period number, $N$, is required to maintain sufficient reflectivity. ${ }^{45}$ Models such as the one described in this work may prove beneficial for the determination of optimal parameters prior to fabrication or experimental characterization of PC structures for optical applications.

\section{Finite Difference Frequency Domain Model}

Next, the FDFD method was used to get a complete plot of the photonic band structure for a 2-D square lattice of dielectric cylinders in a matrix with a different dielectric constant and to compare these results with the above model. As is often done for the TM mode in PC calculations, 


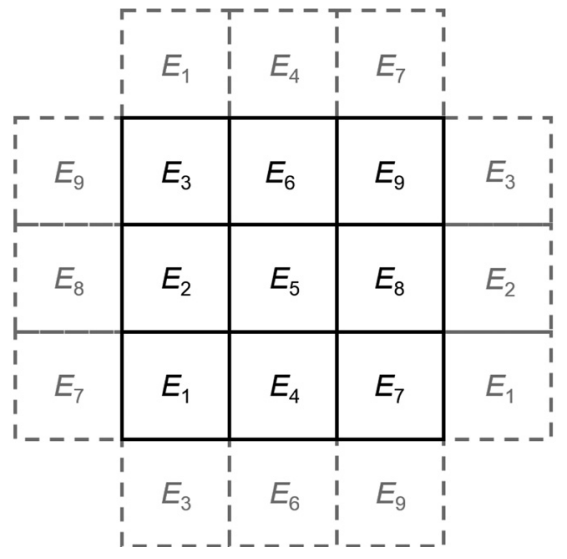

Fig. 5 The square lattice shown for a $3 \times 3$ periodic mesh with the electric field at each position labeled $E_{n}$.

we begin with Eq. (1) (directly derived from Maxwell's equations), as it is dependent only on the electric field. ${ }^{32,46,49}$ The solutions of this eigenvalue equation provide the photonic band structures for the desired crystal and light parameters:

$$
\nabla \times \nabla \times \mathbf{E}(\mathbf{r})=\left(\frac{\omega}{c}\right)^{2} \varepsilon_{r}(\mathbf{r}) \mathbf{E}(\mathbf{r}) .
$$

Equation (1) is an eigenvalue problem that was solved using the FDFD method. ${ }^{49}$ This was accomplished by first setting up a mesh along the desired geometry and calculating the relevant $k$-vectors along the geometry's irreducible Brillouin zone.

To begin, Eq. (1) is simplified using the curl of curl identity:

$$
\nabla[\nabla \cdot \mathbf{E}(\mathbf{r})]-\nabla^{2} \mathbf{E}(\mathbf{r})=\left(\frac{\omega}{c}\right)^{2} \varepsilon_{r}(\mathbf{r}) \mathbf{E}(\mathbf{r})
$$

We assume that there are no free charges, which demands that

$$
\nabla \cdot \mathbf{E}(\mathbf{r})=0 .
$$

Equation (2) now simplifies to

$$
-\nabla^{2} \mathbf{E}(\mathbf{r})=\left(\frac{\omega}{c}\right)^{2} \varepsilon_{r}(\mathbf{r}) \mathbf{E}(\mathbf{r})
$$

Expressing Eq. (3) in terms of second order central finite differences gives the following:

$$
-\left(\frac{E_{i+1, j}^{x}-2 E_{i, j}^{x}+E_{i-1, j}^{x}}{h_{x}^{2}}+\frac{E_{i, j+1}^{y}-2 E_{i, j}^{y}+E_{i, j-1}^{x}}{h_{y}^{2}}, 0\right)=\left(\frac{\omega}{c}\right)^{2} \varepsilon_{r i, j} E_{i, j}\left(E_{i, j}^{x}, E_{i, j}^{y}, 0\right),
$$

where $E^{x}$ and $E^{y}$ are the electric field vectors along $x$ and $y$, and $h_{x}$ and $h_{y}$ are the physical distances between the mesh elements along the $x$ and $y$ axis, respectively. Equation (4) is fully decoupled; that is, changes in $E_{x}$ happen only along $i$ ( $x$-axis), and changes in $E_{y}$ happen only along $j$ (y-axis). This allows it to be rewritten as

$$
-\left(\frac{E_{i+1, j}-2 E_{i, j}+E_{i-1, j}}{h_{x}^{2}}+\frac{E_{i, j+1}-2 E_{i, j}+E_{i, j-1}}{h_{y}^{2}}\right)=\left(\frac{\omega}{c}\right)^{2} \varepsilon_{r i, j} E_{i, j} .
$$

To solve this eigenvalue problem, a mesh is generated along a unit cell of the PC, with $i, j$ values corresponding to mesh elements. Figure 5 shows an example of the $n \times n$ mesh in which the electric field at each position is labeled $E_{n}$. In the finite differences equation, the field at a given 
position, $E_{i, j}$, corresponds to one position, $E_{n}$. The $E_{i \pm 1}$ and $E_{j \pm 1}$ terms correspond to adjacent mesh cells in the lattice. Elements within the unit cell are shown as bounded by solid lines and those within adjacent cells are represented by dashed lines. The Appendix further discusses the mesh elements of the unit cell, as well as the required mesh size at which calculated bands converge to a common value.

The matrix multiplying the electric field eigenvector on the left side of Eq. (5) takes into account the connections between each mesh element and its neighboring elements. Thus, in Fig. 5, $E_{1}$ connects to $E_{2}$ and $E_{4}$ within the same unit cell and connects to $E_{3}$ and $E_{7}$ in adjacent unit cells. Periodic boundary conditions are therefore applied to the resulting matrix in the appropriate positions to account for these intercell connections between the edge mesh elements. This requires the use of imaginary exponential terms, $e^{ \pm \mathbf{i} \cdot \mathbf{a}_{1}}$, for periodic Bloch waves in a periodic crystal. Bloch's theorem maintains that all of the waves capable of propagating within the crystal will do so periodically such that they will exist for $k$-values within the first Brillouin zone of the reciprocal lattice. Appending this exponential to the necessary terms conforms to this formulation and is necessary since only one unit cell is contained in the model geometry. The $k \cdot a$ term within the exponential refers to the component of the wave vector $(k)$ along the direction of the basis vector $(a)$ corresponding to the direction of the connection between mesh points. In the square case, $a_{1}$ is along the $i$ direction and $a_{2}$ is along the $j$ direction. These correspond to $x$ - and $y$-directions, respectively.

Once the eigenvalues are calculated, they are multiplied by $c^{2}$ and square-rooted to isolate the eigenmodes, $\omega$, which are then normalized. The 2-D photonic band structure was calculated for the TM mode of the square lattice GaAs model and is plotted in Figs. 6(a) and 6(b) next to the reflection spectra solution [Fig. 6(c)]. In both the FDFD and reflection models in Fig. 6, the dielectric constant was set to one for air and 11.8 for GaAs. Figure 6(a) also shows the real and reciprocal space lattices used in the model. The photonic band structure result was found to closely match the reflection spectrum as well as agreeing with existing results. ${ }^{46}$

Last, due to the complexity of the FDFD model, constant material properties were implemented. Thus, the dielectric function of the materials in the model was constant instead of being wavelength dependent. As shown in Fig. 6, the FDFD band gap results match very well with the reflection spectrum from the other model in which constant material properties were also used. In physical reality, instead of being constant, the dielectric function depends on the wavelength

(a)
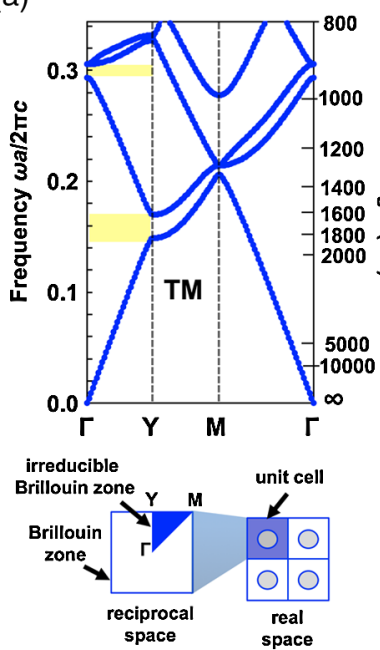

(b)

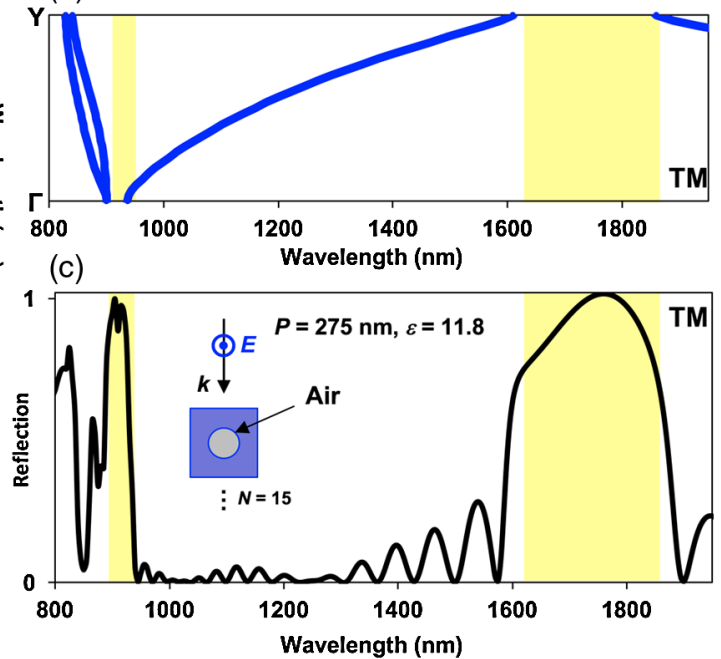

Fig. 6 (a) The photonic band structure calculated through the FDFD model, as well as the simulated unit cell in real space and the corresponding Brillouin zone and irreducible Brillouin zone in reciprocal space, is shown. Band structure is for square lattice with air holes in GaAs and with TM polarization. The dielectric constant here equals 11.8. The (b) band structure and (c) reflection spectrum corresponding to normal incident light parallel to the square lattice are plotted as a function of wavelength for the parameters shown: air holes in GaAs, TM polarization, $P=275 \mathrm{~nm}$. The spectral overlap of the bandgaps in (b) and the reflection peaks in (c) is highlighted. 

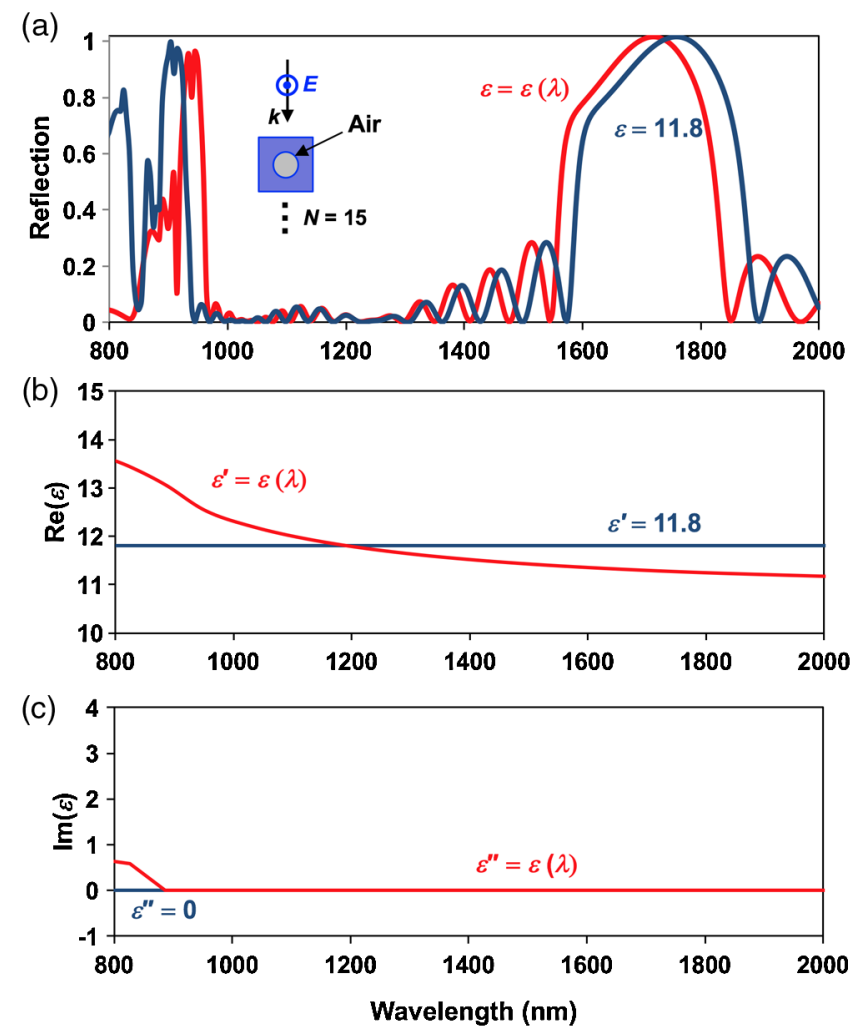

Fig. 7 (a) Plot of reflection spectra for a model with dielectric function $(\varepsilon)$ as a function of wavelength (red) and with a constant dielectric value (blue). $P=275 \mathrm{~nm}$. The (b) real and (c) imaginary parts of the dielectric function for the variable (red) and constant (blue) cases.

of the incident light. For this reason, reflection spectra were calculated for a dielectric constant, $\varepsilon=11.8$, and for a dielectric as a function of wavelength, $\varepsilon(\lambda){ }^{44}$ The resulting spectra are shown in Fig. 7(a) for the wavelength range plotted in previous figures with the constant permittivity shown in blue and variable dielectric function in red. The values of the real $\left(\varepsilon^{\prime}\right)$ and imaginary $\left(\varepsilon^{\prime \prime}\right)$ parts of the dielectric function were plotted versus wavelength as well, as shown in Figs. 7(b) and 7(c), respectively. For a wavelength-dependent dielectric function, $\varepsilon^{\prime}$ continues to vary, albeit close to the constant value of 11.8, as demonstrated in Fig. 7(b). The imaginary part is zero for a constant permittivity and quickly approaches zero for $\varepsilon(\lambda)$. The larger discrepancy between the reflection spectra for wavelengths from 800 to $1000 \mathrm{~nm}$, as compared to the 1000 to $2000 \mathrm{~nm}$ range, is due to the difference between values of $\varepsilon^{\prime \prime}$ for $\varepsilon(\lambda)$ and $\varepsilon=11.8$. See the Appendix for results calculated for air holes in $\mathrm{Si}$ and $\mathrm{SiO}_{2}$ surrounding media.

\section{Conclusion and Discussion}

A compact model was designed to evaluate the reflection of a 2-D square lattice PC with a finite number of periods, $N$, under different conditions. The results showed precisely how the reflectivity changes with $N$. With the reflection model, the effects of using both a constant and variable permittivity were studied. Results reveal the potential errors resulting from using a constant dielectric function in pursuit of the most accurate calculations of photonic band structures. A FDFD model was used to calculate the full TM mode photonic band structure for comparison with the reflection model. The FDFD calculated photonic bandgaps align well with the reflection bands determined through the reflection model. Variations of the model introduced here can also be used to simulate a hexagonal lattice, calculate transmission spectra, and investigate various incident angles. Utilizing this type of model will help facilitate advances in the development of PC technologies. 


\section{Appendix}

Additional materials, $\mathrm{Si}$ and $\mathrm{SiO}_{2}$, were studied with the reflection model as well. Here, models were established to calculate the reflection spectra for air holes in surrounding media of each material for the TM mode with $N=15$, and wavelength-dependent dielectric functions were implemented. ${ }^{50,51}$ Figures 8 (a) and 8 (b) show the results of varying $P$ for each material. As was the case for air holes in GaAs, $\lambda_{\text {peak }}$, the wavelength of the reflection peak is redshifted for increasing $P$. A comparison between the peak shifts for each material is shown in Fig. 8(c). The plot shows the values for $\lambda_{\text {peak }}$ versus $P$ for each material. Comparing the peak widths in Figs. 8(a) and 8(b), the $\mathrm{SiO}_{2}$ peaks are clearly narrower than those calculated for $\mathrm{Si}$.

In order to illustrate the convergence of the FDFD model for a sufficient number of mesh elements in the unit cell, the plot shown in Fig. 9 was created. The plot was created by taking the wavelength values of the first and second bands at the $Y$ point in reciprocal space as a function of mesh elements in one row of the unit cell. This was done for $P=275 \mathrm{~nm}$ with GaAs $(\varepsilon=11.8)$. The plot demonstrates convergence for a unit row containing 25 or more mesh elements.

(a)

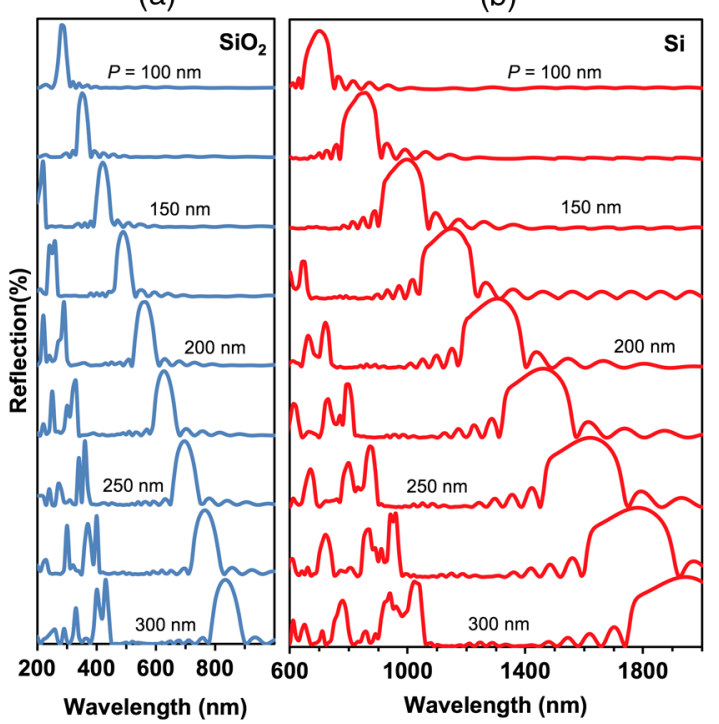

(c)

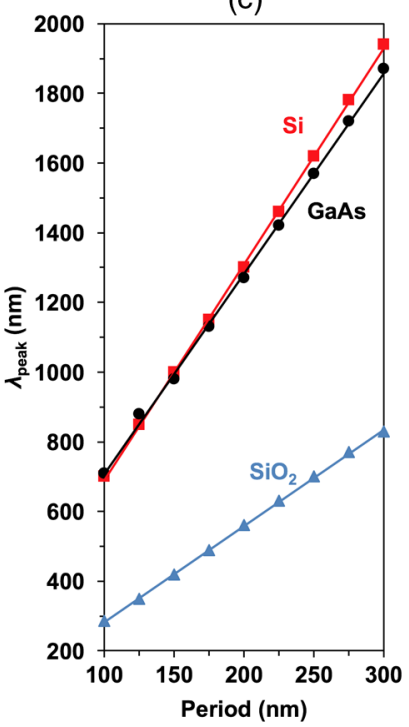

Fig. 8 Reflection spectra for $N=15$ varying PC period widths $(P)$, TM polarization, and air holes in (a) $\mathrm{SiO}_{2}$ and (b) Si surrounding media. (c) Plot of the wavelength at which each reflection peak occurs, $\lambda_{\text {peak }}$, for each material.

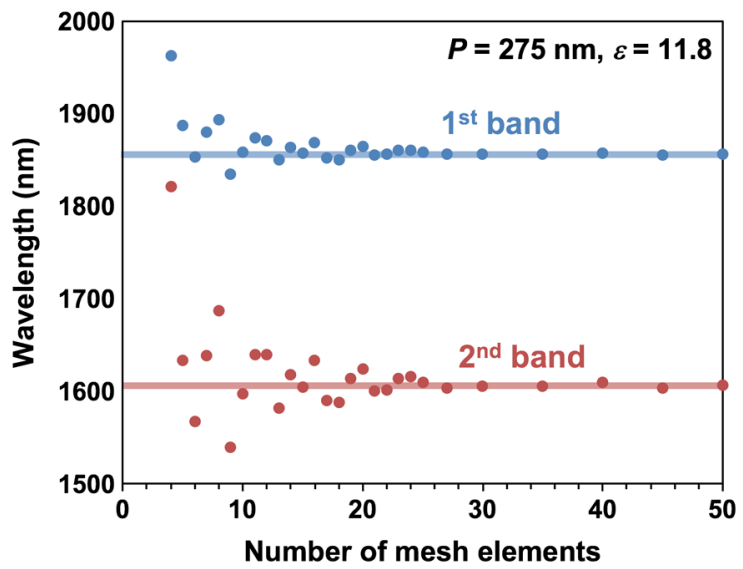

Fig. 9 Convergence test results for the first and second bands showing their wavelength values at the $Y$ point in reciprocal space for different numbers of mesh elements along one direction in the unit cell. 
Thus, in the interest of accuracy, a unit cell of $30 \times 30$ mesh elements was utilized for the FDFD calculations performed to create Figs. 6(a) and 6(b).

\section{Acknowledgments}

Authors would like to acknowledge the help of Salvador Barraza-Lopez through many useful conversations and discussions. Also, we want to recognize Paul Millett for helping to inspire the motivation for this research project. S.B. has received support from the SPIE Optics and Photonics Education Scholarship and the Doctoral Academy Fellowship. J.M. has received support through the George D. Lingelbach Memorial Scholarship and the Student Undergraduate Research Fellowship (SURF) provided by the Arkansas Department of Higher Education. J.H. has received support from the Arkansas Biosciences Institute and from the Provost's Collaborative Research Grant funded by the Office of the Provost at University of Arkansas.

\section{References}

1. L. Marko, Y. Tomoyuki, and S. Axel, "Low-threshold photonic crystal laser," Appl. Phys. Lett. 81(15), 2680 (2002).

2. I. Schnitzer et al., "The single-mode light-emitting-diode," Nano ASI 308, 369-378 (1993).

3. G. Shambat et al., "Ultrafast direct modulation of a single-mode photonic crystal nanocavity light-emitting diode," Nat. Commun. 2, 539 (2011).

4. V. Berger, "Nonlinear photonic crystals," Phys. Rev. Lett. 81, 4136-4139 (1998).

5. A. Kavokin et al., "Photonic Bloch oscillations in laterally confined Bragg mirrors," Phys. Rev. B 61, 4413-4416 (2000).

6. B. Bakir et al., "Surface-emitting microlaser combining two-dimensional photonic crystal membrane and vertical Bragg mirror," Appl. Phys. Lett. 88(8) (2006).

7. M. Qiu et al., "Photonic crystal optical filter based on contra-directional waveguide coupling," Appl. Phys. Lett. 83(5121) (2003).

8. M. Qiu, "Ultra-compact optical filter in two-dimensional photonic crystal," Electron. Lett. 40(9), 539-540 (2004).

9. G. Shambat et al., "Photonic crystal filters for multi-band optical filtering on a monolithic substrate," J. Nanophotonics 3, 031506 (2009).

10. E. R. Brown and E. R. Parker, "Radiation properties of a planar antenna on a photonic crystal," Opt. Soc. 10(2), 404 (1993).

11. T. Liu et al., "Design of a compact photonic-crystal-based polarizing beam splitter," IEEE Photonics Technol. Lett. 17(7), 1435-1437 (2005).

12. S. G. Johnson et al., "Guided modes in photonic crystal slabs," Phys. Rev. B 60(8), 5751-5758 (1999).

13. T. R. Woliński et al., "Polarization effects in photonic liquid crystal fibers," Meas. Sci. Technol. 18(10), 3061-3069 (2007).

14. P. M. Bell et al., "A program for calculating photonic band structures and transmission coefficients of complex structures," Comput. Phys. Commun. 85(2), 306-322 (1995).

15. M. M. Sigalas et al., "Theoretical investigation of off-plane propagation of electromagnetic waves in two-dimensional photonic crystals," Phys. Rev. B 58(11), 6791-6794 (1998).

16. B. P. Hiett et al., "Application of finite element methods to photonic crystal modelling," IEE Proc.-Sci. Meas. Technol. 149(5), 293-296 (2002).

17. W. Axmann and P. Kuchment, "An efficient finite element method for computing spectra of photonic and acoustic band-gap materials," J. Comput. Phys. 150(2), 468-481 (1999).

18. A. Peyrilloux et al., "Comparison between the finite element method, the localized function method and a novel equivalent averaged index method for modelling photonic crystal fibres," J. Opt. Pure Appl. Opt. 4(3), 257-262 (2002).

19. D. C. Dobson, "An efficient method for band structure calculations in 2D photonic crystals," J. Comput. Phys. 149(2), 363-376 (1999).

20. D. C. Dobson, J. Gopalakrishnan, and J. E. Pasciak, "An efficient method for band structure calculations in 3D photonic crystals," J. Comput. Phys. 161(2), 668-679 (2000). 
21. T. Baba et al., "Light propagation characteristics of straight single-line-defect waveguides in photonic crystal slabs fabricated into a silicon-on-insulator substrate," IEEE J. Quantum Electron. 38(7), 743-752 (2002).

22. A. Chutinan and S. Noda, "Waveguides and waveguide bends in two-dimensional photonic crystal slabs," Phys. Rev. B 62(7), 4488-4492 (2000).

23. M. Qiu and S. He, "A nonorthogonal finite-difference time-domain method for computing the band structure of a two-dimensional photonic crystal with dielectric and metallic inclusions," J. Appl. Phys. 87(12), 8268-8275 (2000).

24. M. Qiu and S. He, "FDTD algorithm for computing the off-plane band structure in a twodimensional photonic crystal with dielectric or metallic inclusions," Phys. Lett. A 278(6), 348-354 (2001).

25. L. Wu and S. He, "Revised finite-difference time-domain algorithm in a nonorthogonal coordinate system and its application to the computation of the band structure of a photonic crystal," J. Appl. Phys. 91(10), 6499-6506 (2002).

26. Y. Cao, Z. Hou, and Y. Liu, "Finite difference time domain method for band-structure calculations of two-dimensional phononic crystals," Solid State Commun. 132(8), 539-543 (2004).

27. M. Qiu, "Analysis of guided modes in photonic crystal fibers using the finite-difference time-domain method," Microwave Opt. Technol. Lett. 30(5), 327-330 (2001).

28. J.-H. Sun and T.-T. Wu, "Propagation of surface acoustic waves through sharply bent twodimensional phononic crystal waveguides using a finite-difference time-domain method," Phys. Rev. B 74(17), 174305 (2006).

29. H. Y. D. Yang, "Finite difference analysis of 2-D photonic crystals," IEEE Trans. Microwave Theory Tech. 44(12), 2688-2695 (1996).

30. C. Yu and H. Chang, "Compact finite-difference frequency-domain method for the analysis of two-dimensional photonic crystals," Opt. Express 12(7), 1397 (2004).

31. S. Guo et al., "Photonic band gap analysis using finite-difference frequency-domain method," Opt. Express 12(8), 1741 (2004).

32. C. Van Der Mee, S. Seatzu, and P. Contu, "Fast and effective finite difference method for 2D photonic crystals," Commun. Appl. Ind. Math. 2(2), 1-18 (2011).

33. R. D. Meade et al., "Accurate theoretical analysis of photonic band-gap materials," Phys. Rev. B 48(11), 8434-8437 (1993).

34. K. M. Ho, C. T. Chan, and C. M. Soukoulis, "Existence of a photonic gap in periodic dielectric structures," Phys. Rev. Lett. 65(25), 3152-3155 (1990).

35. C. T. Chan, Q. L. Yu, and K. M. Ho, "Order-N spectral method for electromagnetic waves," Phys. Rev. B 51(23), 16635-16642 (1995).

36. S. Xiao, L. Shen, and S. He, "A plane-wave expansion method based on the effective medium theory for calculating the band structure of a two-dimensional photonic crystal," Phys. Lett. A 313(1-2), 132-138 (2003).

37. A. G. Hanif, T. Arima, and T. Uno, "Finite-Difference frequency-domain algorithm for band-diagram calculation of 2-D photonic crystals composed of debye-type dispersive materials," IEEE Antennas Wireless Propag. Lett. 11, 41-44 (2012).

38. A. Mekis et al., "High transmission through sharp bends in photonic crystal waveguides," Phys. Rev. Lett. 77(18), 3787-3790 (1996).

39. H.-Y. Ryu, M. Notomi, and Y.-H. Lee, "Finite-difference time-domain investigation of band-edge resonant modes in finite-size two-dimensional photonic crystal slab," Phys. Rev. B 68(4), 45209 (2003).

40. S. Yamada et al., "Simulation of optical pulse propagation in a two-dimensional photonic crystal waveguide using a high accuracy finite-difference time-domain algorithm," J. Appl. Phys. 93(4), 1859-1864 (2003).

41. Z.-Y. Li and K.-M. Ho, "Light propagation in semi-infinite photonic crystals and related waveguide structures," Phys. Rev. B 68(15), 155101 (2003).

42. Y.-C. Hsue and T.-J. Yang, "Applying a modified plane-wave expansion method to the calculations of transmittivity and reflectivity of a semi-infinite photonic crystal," Phys. Rev. E 70(1), 16706 (2004). 
43. W. J. Kim and J. D. O'Brien, "Optimization of a two-dimensional photonic-crystal waveguide branch by simulated annealing and the finite-element method," J. Opt. Soc. Am. B 21(2), 289 (2004).

44. J. B. Theeten, D. E. Aspnes, and R. P. H. Chang, "A new resonant ellipsometric technique for characterizing the interface between GaAs and its plasma-grown oxide," J. Appl. Phys. 49(12), 6097-6102 (1978).

45. F. L. Pedrotti and L. S. Pedrotti, Introduction to Optics, 2nd ed., Prentice Hall, New Jersey (1993).

46. J. D. Joannopoulos et al., Photonic Crystals: Molding the Flow of Light, 2nd ed., Princeton University Press, Princeton (2008).

47. I. Abdulhalim, "Effect of the number of sublayers on axial optics of anisotropic helical structures," Appl. Opt. 47(16), 3002 (2008).

48. S. K. Tripathy, "Refractive indices of semiconductors from energy gaps," Opt. Mater. 46, 240-246 (2015).

49. M. Sarollahi et al., "The significance of the number of periods and period size in 2D photonic crystal waveguides," Proc. SPIE 9556, 95561B (2015).

50. M. A. Green and M. J. Keevers, "Optical properties of intrinsic silicon at $300 \mathrm{~K}$," Prog. Photovoltaic Res. Appl. 3(3), 189-192 (1995).

51. L. Gao, F. Lemarchand, and M. Lequime, "Refractive index determination of $\mathrm{SiO}_{2}$ layer in the UV/Vis/NIR range: spectrophotometric reverse engineering on single and bi-layer designs," J. Eur. Opt. Soc. Rapid Publ. 8, 130101 (2013).

Mirsaeid Sarollahi is a PhD student in electrical engineering at the University of Arkansas studying solar cell efficiency. He received his bachelor of science degree in physics from Zanjan University in Iran and his master's in physics from Azad University (Iran). He worked with fiber optic design at the Telecommunication Company of Zanjan, Iran. He performed the present work with Dr. Herzog at the University of Arkansas.

Stephen J. Bauman is currently a $\mathrm{PhD}$ candidate in the microelectronics and photonics graduate program at the University of Arkansas. He studies experimental and theoretical plasmonics and other areas of nano-optics, such as photonic crystals. He received his dual BS degrees from Southeast Missouri State University in physics and engineering physics and received his MS in microelectronics and photonics from the University of Arkansas. He is actively involved with SPIE.

Jonathan Mishler joined the Herzog Lab in 2013. He graduated from the University of Arkansas with a bachelor's in physics. He then became a postbaccalaureate at the National Institutes of Health in Bethesda, Maryland. In fall 2016, he began graduate school at the University of Washington (Seattle) in the Department of Bioengineering.

Joseph B. Herzog is an assistant professor in the Department of Physics at the University of Arkansas. He received his BS degree from Louisiana State University and his MS and $\mathrm{PhD}$ degrees from the University of Notre Dame. He was a Welch postdoctoral research associate at Rice University. His research focuses on nano-optics including plasmonics and photonic crystals. He is an active member of SPIE, serving on various committees. 